\title{
An Efficient Approach in Detecting Water Leakage in Superstructure
}

\author{
Mrs Kalaivani $\mathbf{M}^{1}$, Dr Jeyalakshmi $\mathbf{C}^{2}$, Mrs Dharini $\mathbf{D}^{3}$ \\ Assistant Professor, Department of ECE, K Ramakrishnan College of Engineering, Trichy, India ${ }^{1,3}$ \\ Associate Professor, Department of ECE, K Ramakrishnan College of Engineering, Trichy, India ${ }^{2}$
}

\begin{abstract}
Automatic leakage detection and maintenance of superstructure is a challenging task in current civil frame work. Current detection standards require a detector to locate the target architecture location and visually appraise the superstructure condition. When the target location is impassable, lorgnette must be used to recognize the defects. This paper proposes a labour-intensive approach, by means of image processing which uses a robotic system to monitor and detect the superstructure frequently and execute independent leakage detection. This paper uses fuzzy KNN algorithm to detect water leakage in the building wall.
\end{abstract}

Keywords: Fuzzy KNN Algorithm; Image Processing; Labour Intensive Approach; Leakage Detection

\section{INTRODUCTION}

One of the elemental demands in case of all buildings structure is that it should be dry as long as possible. If the building structure has not satisfied the above condition, then it shows that the building may be unsafe to live. The entry of water or moisture in the building wall is known as leakage. Leakage in the building walls is most common and it is most important to take necessary precaution steps to avoid damage over the building. Recently the detection of leakage water from underground pipelines was identified using Thermography Infra-Red camera [1]. Now days, even a simple Infra-Red cameras were performing well to analyse the moisture. Because of evaporative cooling, the Infra-Red camera can able to balance the argumentative moisture audit quickly \& non-destructively. By this it is easy to pinpoint the exact location and size of the moisture affected region [2]. The River Project in Libya, by considering the level of dryness and the dimensions, it shows that by using infrared camera it is simple to detect the leakages in water pipes [3]. Here, we proposed a water leakage system by using Digital Image Processing, on the basis of non destructive test. The probability of using certain image processing techniques to recognize worsening in building superstructures was widely recognized by the superior researches. This paper presents a new leakage detection technique, by using simple digital camera. The exact condition of the wall is identified by using the fuzzy KNN algorithm [4]. By analysing the result, we can determine how long the wall will exist and the total amount of water content inside the wall.

\section{IMAGE PROCESSING}

In Image or speech signal the pre-processing is the first step which removes the unwanted distortions and enhances the image or speech features so that it will be easy for further processing [5]. The various stages in pre-processing in this proposed method are explained as follows:

A. Normalizing: Normalization is the process in which changes in the intensity values of the pixels were made. Normalization is also termed as contrast stretching or histogram stretching. The linear normalization of gray Scale digital image can be performed according to the formula ,

$$
\mathrm{I}_{\mathrm{N}}=(\mathrm{I}-\mathrm{Min}) \frac{\text { newMax }- \text { newMin }}{\operatorname{Max}-\operatorname{Min}}+\text { newMin }
$$

B. Filtering: Filtering technique is used to enhance the image by removing unnecessary information from the image [9]. By filtering, we can remove noises from the image. In the Digital image processing, filters were used for smoothing, enhancing and detecting edges in the image. The image can be filtered in both frequency domain or in the spatial domain.

C. Histogram Modification: Histogram plays an important role in image enhancement. By means of Histogram modification, one can analyze and easily modify the characteristics of an image. An example of Histogram modification is the Histogram Equalization. Histogram equalization is a technique by which image intensities were adjusted to enhance contrast. 
Vol. 8, Issue 5, May 2019

\section{IMAGE SEGMENTATION}

The process of partitioning a digital image into a number of segments is known as Image Segmentation. The aim of this process is to convert the image into some useful meaningful one and for easy analysis. It splits entire image into a group of pixels in which each pixel contains some meaningful information.

A. Thresholding: In thresholding method, the foreground objects are separated from background images. The objects are lighter than the background. Depending upon the selection of threshold value, there are several types under the thresholding method, which are Global thresholding, Variable thresholding, Multiple thresholding. The advantage over this method is that it was simple to implement whereas best suitable for document purpose and not suitable for digital image where there is no assurance for object coherency.

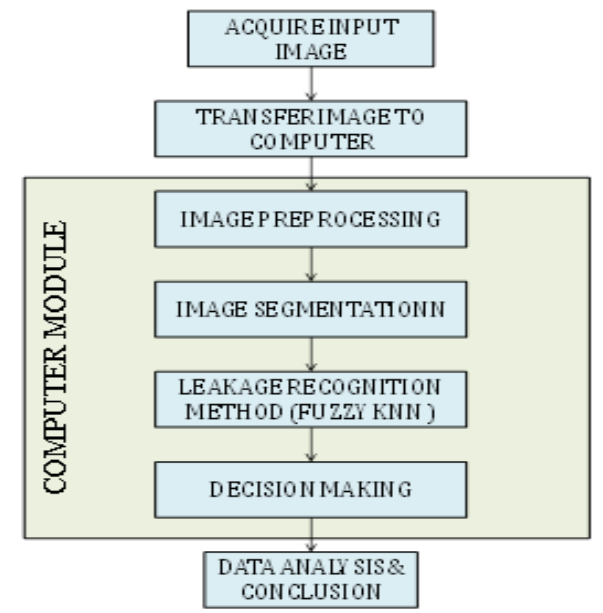

Figure 1 Proposed Image Processing Model

B. Edge Based Segmentation: The pixel connected to form the boundary of the image is known as edge [8]. The edge is the derivative function of the image. Edge based image segmentation process was best suitable for structural techniques as it is easy to find the Ramp edge, Step edge, Roof edge and Spike edge.

C. Region based segmentation: This method is based on splitting the similar pixel values and dissimilar pixel values into separate group. Here there are two types, Region growing method and split \& merge method. The main advantage of this type is that the connected regions are guaranteed \& hence it reduces the problems during merging [6].

D. Clustering Based Segmentation: In this method, the pixels having similar values are grouped together.

\section{LEAKAGE RECOGNITION USING FUZZY KNN ALGORITHM}

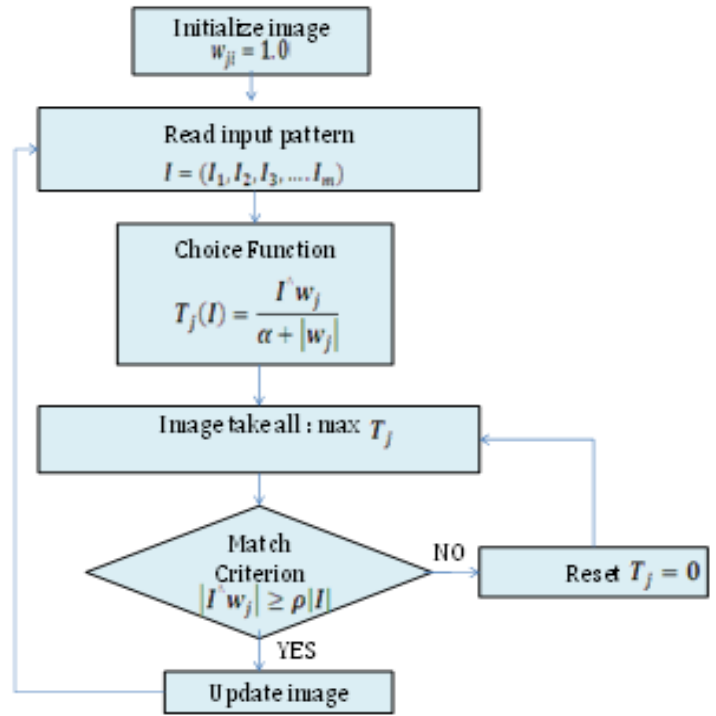

Figure 2 Fuzzy KNN Flowchart 
Vol. 8, Issue 5, May 2019

The KNN classifier is a non-parametric and instance-based learning algorithm. It is a simple algorithm that accumulates all available data's and classifies new data based on a similarity measure. The k-Nearest Neighbors (KNN) algorithm [7] is mainly used to solve classification problems. Using this algorithm one can easily interpret the output, the time needed can be reduced with very low power consumption. The classification is based on the similarity of the training data.

\section{RESULTS \& DISCUSSION}

The image captured by the infrared camera of a building wall is taken as input and scanning of the image take place for the first process of analyzing of the image which remove the noise of the image thus gives the image which we need for analyzing which undergoes the filtering process and produce the particular image with water leakage and based on the level of damage they detect the condition of the wall and depth of the water inside the wall. Image report has been classified according to damage as major, minor and healthy thus we can easily make the change to repair the building and thus reduce the production cost and repair cost because it is the non-destructive test. Minor damage is about the building having the small amount of water leakage inside that can be detected and reported as minor level of repair is required and these outputs were shown below in the output figures. In the case of the healthy image the regular damage caused by the atmosphere can be detected and thus it is not affected by the water leakage and they do not need the repair of the building in this condition thus the report generated is about nil damage and no need of building repair is needed.

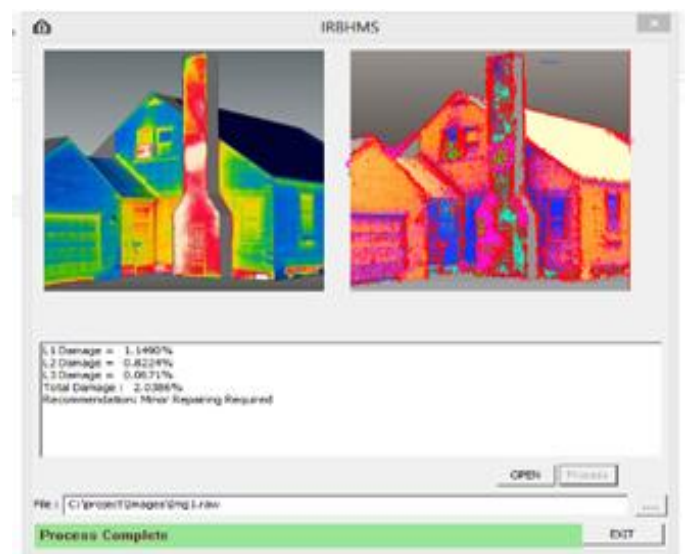

Figure 3 Image showing Minor Repair required

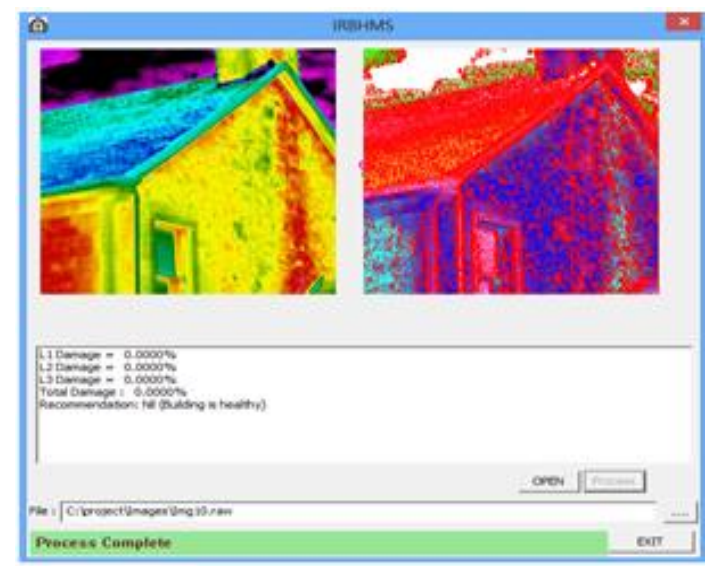

Figure 4 Image of a Healthy Building

VI. CONCLUSION

In this paper, a new method for water detection inside the building is presented. This new method is based on the digital image processing. By using fuzzy KNN algorithms an efficient detection of building and damage Classification method was constructed. The Proposed method is developed for diagnosing the water inside the building without destruction. This system performs the diagnosis in multiple phases. First phase of diagnosis is image processing and it continue with the scanning and the second phase is that image pre-processing which includes the analyzing, filtering and normalizing then the process of analyzing is particularly differentiated into three damage stages as L1, L2, L3 .damages were 
Vol. 8, Issue 5, May 2019

classified as minor damage, major damage and acute damage thus the final output report is displayed in the output report box. Hence from the result of proposed method we can easily make the change to repair the building and thus reduce the production cost and repair cost because it is the non-destructive test.

\section{REFERENCES}

[1]. Cheng, H. D., Chen, J., Glazier, C., and Hu, Y. G. (1999). Novel approach to pavement cracking detection based on fuzzy set theory, Journal of Computing in Civil Engineering, 13(4) 270-280.

[2]. Jeyalakshmi, C Murugeshwari, B Karthick, M, "HMM and K-NN based Automatic Musical Instrument Recognition" Proceedings of $2^{\text {nd }}$ International conference on I-SMAC (IoT in Social, Mobile, Analytics and Cloud,2018).

[3]. D.Dharini, A.Revathy, M.Kalaivani, Contrast Of Gaussian Mixture Model And Clustering Algorithm For Singer Identification" 2018,Proceedings of International Conference On Computer Communication And Informatics (ICCCI -2018), Jan. 04 - 06, 2018.

[4]. Bubaker Shakmak; Amin Al-Habaibeh. "Detection of water leakage in buried pipes using infrared technology; A comparative study of using high and low resolution infrared cameras for evaluating distant remote detection" 2015 IEEE Jordan Conference on Applied Electrical Engineering and Computing Technologies (AEECT)

[5]. Revathi, A Jeyalakshmi, C 2017, 'A challenging task in recognizing the speech of the hearing impaired using normal hearing models in classical Tamil language', Journal of Engineering research, vol.5 no.2, pp.110-128

[6]. Gerhardus H. Koch, Michiel P.H. Brongers, and Neil G. Thompson, 2017, 'Corrosion costs and preventive strategies in the United States', NACE International, 2017, August 14.

[7]. Revathi A Jeyalakshmi, C 2018, "Emotions recognition: different sets of features and models", International Journal of Speech Technology, https://doi.org/10.1007/s10772-018-9533-6.

[8]. R Preethi Rajaiah, R John Britto 2014, Optic disc boundary detection and cup segmentation for prediction of glaucoma, International Journal of Science, Engineering and Technology Research (IJSETR), vol.3, issue 10, pp. 2665-2672.

[9]. A Revathi, C Jeyalakshmi, 2017, Robust speech recognition in noisy environment using perceptual features and adaptive filters, Proceedings of 2nd International Conference on Communication and Electronics Systems (ICCES), pp. 692-696.

\section{BIOGRAPHIES}

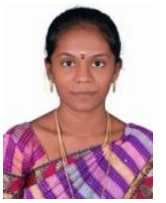

Mrs M. Kalaivani received her B.E degree with distinction in Electronics and Communication Engineering from Ponnaiyah Ramajayam College of Engineering and Technology, Tanjore, M.E degree in Communication Systems from Dhanalakshmi Srinivasan Engineering College, Perambalur. She is currently working as Assistant Professor at K.Ramakrishnan College of Engineering, Tiruchirappalli. She is Member of national Professional Society ISTE.

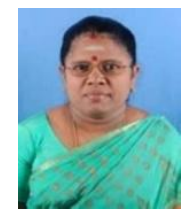

Dr.C.Jeyalakshmi received B.E degree in Electronics and Communication Engineering from Bharathidasan University in 2002 and M.E. degree in Communication systems from Anna University, Chennai in 2008. She served as a faculty for 11 years in the Department of ECE, Trichy Engineering college, Tamilnadu. Currently she has been with K.Ramakrishnan college of Engg., where she is working as Associate professor in ECE dept. She has obtained PhD degree from Anna University, Chennai in the field of Speech recognition of hearing impaired people in 2015. Her research interest also includes speech processing, Image processing, Neural networks, RF and Microwave Engineering. She has published 25 papers in Reputed International journals and presented papers in more than 10 International Conferences.

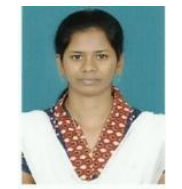

Mrs.D.DHARINI received her B.E degree with distinction in Electronics and Communication Engineering from M.A.M College of Engineering, Tiruchirappalli, M.E degree with University Rank in Communication Systems from Saranathan College of Engineering, Tiruchirappalli. She is Member of national Professional Society ISTE. 